\title{
Conversion from Intermediate Age-Related Macular Degeneration to Geographic Atrophy in a Proxima B Subcohort Using a Multimodal Approach
}

\author{
Steffen Schmitz-Valckenberga, ${ }^{a}$ Martina D. Braun ${ }^{a}$ Sarah Thiele ${ }^{a}$ \\ Daniela Ferrara $^{c}$ Lee Honigberg ${ }^{c}$ Simon S. Gao ${ }^{c}$ Hao Chen ${ }^{c}$ Verena Steffen ${ }^{c}$ \\ Frank G. Holz ${ }^{a}$ Marlene Saßmannshausen ${ }^{a}$ \\ aGRADE Reading Center and Department of Ophthalmology, University of Bonn, Bonn, Germany; bohn A. Moran \\ Eye Center, University of Utah, Salt Lake City, UT, USA; 'Genentech, Inc., South San Francisco, CA, USA
}

\section{Keywords}

Age-related macular degeneration - Geographic atrophy ·

Multimodal imaging $\cdot$ Reticular pseudodrusen

\begin{abstract}
Introduction: This retrospective analysis assessed geographic atrophy (GA) progression in fellow eyes from the Proxima $B$ trial intermediate age-related macular degeneration (iAMD) subcohort using high-resolution multimodal imaging anchored on optical coherence tomography (OCT). Methods: Thirty-two patients from the Proxima B iAMD subcohort were assessed; all had GA with no macular neovascularization (MNV) in the contralateral eye. Imaging data, including color fundus photography, fluorescein angiography, near-infrared reflectance, fundus autofluorescence (FAF), and spectral-domain OCT, were obtained. Features preceding progression/conversion to advanced AMD (drusen, reticular pseudodrusen [RPD], MNV, incomplete/complete retinal pigment epithelium and outer retinal atrophy [iRORA/cRORA]) were assessed. Results: Of 30 fellow eyes with available follow-up images, 12 converted to GA (FAF), 2 converted to $M N V$, and 16 were nonconverters during the review period (median: 17.8 months). iRORA/cRORA features
\end{abstract}

karger@karger.com www.karger.com/oph

Karger $\stackrel{\text { ' }}{5}$

GOPEN ACCESS
(C) 2021 The Author(s)

Published by S. Karger AG, Basel

This is an Open Access article licensed under the Creative Commons Attribution-NonCommercial-4.0 International License (CC BY-NC) (http://www.karger.com/Services/OpenAccessLicense), applicable to the online version of the article only. Usage and distribution for commercial purposes requires written permission. (present in all converters at baseline) were identified on OCT in eyes that progressed to GA. Median time interval from iRORA to CRORA and from CRORA to GA was 7 months each. GA development/progression was either drusen- or RPD-associated ( $n=6$ each). Eyes with baseline RPD showed faster GA progression versus eyes with drusen (1.49 vs. $0.38 \mathrm{~mm}^{2} /$ year). Conclusions: RPD presence was associated with rapid GA lesion enlargement and may provide an early indication of faster $G A$ progression.

(c) 2021 The Author(s) Published by S. Karger AG, Basel

\section{Introduction}

Age-related macular degeneration (AMD) is one of the leading causes of irreversible vision loss in older people in industrial countries $[1,2]$. Progression of disease from intermediate AMD (iAMD) to vision-threatening advanced AMD, which can manifest as either macular neovascularization (MNV) or geographic atrophy (GA) $[3,4]$, is poorly understood [5-7]. GA is characterized by loss of choriocapillaris, retinal pigment epithelium (RPE), and photoreceptors $[5,8]$. Treatment of GA remains a significant unmet medical need, as there are no approved 
therapies to prevent onset of GA secondary to AMD or to halt or slow GA lesion progression [7]. A major challenge is to identify precursor lesions and pathways leading to GA development and lesion growth. Moreover, better understanding of these key aspects appears crucial for defining robust clinical end points for future AMD studies.

High-resolution multimodal imaging potentially allows for identification of GA precursors and detailed characterization of conversion to GA. However, data on development and progression of GA, including early GA lesion growth and course of visual impairment in patients with GA, are scarce $[6,9-12]$. The lampalizumab clinical development program investigated effects of anti-factor D (lampalizumab) in patients with GA secondary to AMD [12]. In parallel with the lampalizumab programs, 2 global, prospective, noninterventional, observational studies (Proxima A and B [12]) were conducted to characterize visual function decline associated with progression of GA secondary to AMD. Proxima A enrolled participants with bilateral GA without MNV in either eye at baseline. Proxima B enrolled patients with unilateral GA with either MNV or iAMD in the fellow eye at baseline and was designed to capture phenotypic variations of GA not captured in Proxima A or other studies in the lampalizumab program [12].

In the present study, we conducted a retrospective exploratory analysis to assess the subgroup in Proxima B with unilateral GA in 1 eye and iAMD in the other eye. Eyes assigned as fellow eyes in Proxima B were the eyes of interest in the present study. The objective of the present analysis was to identify and characterize precursor features for development of GA in the fellow eye iAMD cohort from Proxima $B$ based on multimodal imaging analysis. In eyes that converted from iAMD to GA during follow-up, GA progression rate was also determined.

\section{Materials and Methods}

\section{Proxima B Study Design and Participants}

Primary results of Proxima $B$ have been reported previously [12]. Briefly, Proxima B (NCT02399072) was a global, prospective, observational, noninterventional, natural history study conducted at 53 sites in 11 countries. Proxima B was originally planned to enroll 200 patients, with a follow-up period of 60 months; however, the study was terminated early on November 20, 2017 (last patient visit: January 31, 2018), after the lampalizumab phase III trials did not meet the primary end point of efficacy over sham controls $[12,13]$. Changes in visual function parameters (i.e., bestcorrected visual acuity [BCVA] and low-luminance visual acuity) and imaging (color fundus photography [CFP], fluorescein angiography [FA], near-infrared reflectance [NIR], fundus autofluorescence $[\mathrm{FAF}]$, spectral-domain optical coherence tomography
[SD-OCT]), and anatomic characteristics were followed with comprehensive clinical assessment and multimodal imaging over time. Proxima B was approved by the institutional review board or received ethics committee approval at each participating site (see Statement of Ethics). The study was conducted according to the provisions of the Declaration of Helsinki and Good Clinical Practice Guidelines of the International Conference on Harmonisation of Technical Requirements for Registration of Pharmaceuticals for Human Use, and all applicable local, state, and federal laws. All participants provided informed consent.

In the parent Proxima B study, there were 2 cohorts, namely, (1) fellow eye MNV cohort: GA with no MNV in the study eye, and MNV in the fellow eye with or without GA; and (2) fellow eye iAMD cohort: GA with no MNV in the study eye, and iAMD (i.e., no MNV or GA) in the fellow eye. As mentioned previously, eyes assigned as fellow eyes in Proxima B were the eyes of interest in the present study.

Patients were eligible for enrollment in the parent Proxima B study if they were aged $\geq 50$ years and were able to return for all scheduled visits and examinations. Key eligibility criteria for the study eye in the parent Proxima B study were Early Treatment Diabetic Retinopathy Study BCVA score $\geq 19$ letters (Snellen equivalent 20/400 or better); well-demarcated area(s) of GA secondary to AMD with total lesion size $0.3-17.78 \mathrm{~mm}^{2}$ ( $\sim 0.1-7$ disc areas) or, if multifocal, $\geq 1$ focal lesion $\geq 0.3 \mathrm{~mm}^{2}$, residing completely within the FAF imaging field (field $2-30^{\circ}$, image centered on fovea); perilesional banded or diffuse hyperautofluorescence patterns observed on FAF; and no evidence of prior or active MNV [12].

\section{Imaging Assessments and Outcomes}

For the present analyses, longitudinal multimodal imaging data from both eyes, including confocal scanning laser ophthalmoscopy, SD-OCT, and FAF (obtained at baseline and every 6 months), as well as FA (obtained at baseline), CFP (obtained at baseline and every 12 months), and NIR, were obtained according to standard operational procedures. Imaging data were sent to a central reading center with constant monitoring and feedback on image quality and adherence to imaging procedures. Fundus images of study and fellow eyes at baseline and 6-month assessments were evaluated at the GRADE Reading Center (Bonn, Germany) according to predefined grading criteria, up to an anticipated 60 months and at the early termination visit $\leq 2$ months after the last visit of participants who discontinued before study completion.

Diagnosis and assessment of GA were based on FAF imaging, and other imaging modalities, including NIR, SD-OCT, and FA, were used to confirm key features of the GA lesion and absence of MNV. Foveal involvement in GA was determined by multimodal imaging based on SD-OCT. Change in GA area from baseline as assessed by FAF was a prespecified outcome in Proxima B. GA lesion area was assessed based on semiautomated lesion boundary discrimination using RegionFinder ${ }^{\circledR}$ software (Heidelberg Engineering, Heidelberg, Germany), as previously described [14].

Conversion from iAMD to GA had been previously defined in Proxima B as unifocal or multifocal GA with total area size $\geq 0.15$ $\mathrm{mm}^{2}$ and minimal spot size $\geq 0.05 \mathrm{~mm}^{2}$ (in case of multifocality), as measured by the RegionFinder ${ }^{\circledR}$ software, showing well-demarcated, severely reduced FAF corresponding to outer nuclear layer (ONL) loss and choroidal signal hypertransmission on SD-OCT, and spatially confined to either complete or incomplete depigmentation or other AMD-typical changes. 
Detection of reticular pseudodrusen (RPD) was based on qualitative assessment as part of the original reading center assessment involving 1 junior and 1 senior reader according to the following definitions. Using confocal scanning laser ophthalmoscopy, NIR, and FAF imaging, RPD was characterized as a group of hyperreflective dots, hyporeflective rings with a hyperreflective center, or irregular wriggled patterns (i.e., ribbons); in SD-OCT, $\geq 5$ hyperreflective mounds above the RPE, or "spikes" (lesions breaking through the external limiting membrane $[\mathrm{ELM}]$ ), were required to be present in $\geq 1$ B-scan $[15,16]$. The RPD could then be defined as either "not determinable" (grader was $<90 \%$ certain that RPD was present) or "yes" (grader was $>90 \%$ certain that RPD was present).

Methods and Definitions Used in the Present Retrospective Exploratory Image Analysis

To account for the new definition of various atrophy manifestations recently brought forward by the Classification of Atrophy Meetings (CAM) group $[17,18]$, the present analyses also assessed signs of incomplete or complete RPE and outer retinal atrophy (iRORA/cRORA) in eyes with iAMD at baseline and during the follow-up period. In all eyes where iRORA/cRORA (based on SDOCT) or GA (based on FAF) was identified during the review period, multimodal imaging data from all available time points were retrospectively analyzed for qualitative features. As opposed to the prospective image analysis in the parent Proxima B study, this approach also allowed the retrospective series review of individual eyes, including point-by-point correlation with the site of development of iRORA/cRORA or GA. In this context, follow-up mode, which allowed, at the subsequent visit, placement of single B-scans at the same retinal location as performed at baseline, for SD-OCT imaging was used to analyze evolution of atrophy at any given retinal location over time. The analysis focused on identification of patterns and precursor lesions leading to atrophy, by focusing on 1 atrophic lesion per eye. If $>1$ lesion of manifest atrophy was initially present, the largest of these lesions was selected. Further, we looked at early progression of GA once conversion had occurred, including all areas with manifest atrophy.

iRORA was defined on SD-OCT based on the following criteria: (1) region of signal hypertransmission into the choroid; (2) corresponding zone of attenuation or disruption of the RPE, with or without persistence of basal laminar deposits; and (3) evidence of overlying photoreceptor degeneration, which is subsidence of the inner nuclear layer (INL) and outer plexiform layer (OPL), presence of a hyporeflective wedge in Henle's fiber layer, thinning of the ONL, disruption of the ELM, or disintegrity of the ellipsoid zone (EZ), when these criteria do not meet the definition of cRORA [18]. The CAM group recommended that the term nascent GA (nGA), which had been previously used to describe the above features on SD-OCT, be used more broadly to describe iRORA in the absence of current or prior MNV $[17,18]$. Further, the original definition of nGA does not include the criteria of ELM disruption, EZ disruption, ONL thinning, and attenuation/disruption of the RPE.

cRORA was defined on SD-OCT based on the following criteria: (1) zone of hypertransmission $\geq 250 \mu \mathrm{m}$ in diameter in any lateral dimension; (2) zone of attenuation or disruption of RPE band $\geq 250 \mu \mathrm{m}$; and (3) evidence of overlying photoreceptor degeneration whose features include ONL thinning, ELM loss, and EZ or interdigitation zone loss [17]. Finally, shallow, irregular RPE elevations (SIRE) that have recently been identified as relevant structural OCT signs suggestive of nonexudative MNV [19] were assessed at the baseline visit in the absence of signs of exudation (i.e., intraretinal, subretinal, or sub-RPE fluid; exudates; hemorrhages). As previously described, SIRE was used as a collective term to describe the features of the double-layer sign, or RPE elevation from Bruch's membrane, in the presence of nonexudative MNV. These features include RPE elevations $\geq 1,000 \mu \mathrm{m}$ in diameter, with a maximum elevation height $<100 \mu \mathrm{m}$ and nonhomogenous internal reflectivity [19].

\section{Statistical Methods}

Participants who had baseline assessments and $\geq 1$ post-baseline assessment were included in the analysis. Descriptive statistics are presented for demographic and baseline characteristics, and no statistical analyses were performed given the small sample size. Progression of GA lesion size was determined by subtracting total lesion size measured on FAF at the last available visit from the earliest visit with GA manifestation and extrapolated to yearly growth rate by dividing the difference by number of days between these visits and multiplying by 365.25 . In addition to absolute GA area progression, square-root transformations of lesion size progression were employed.

\section{Results}

\section{Patients and Characteristics at Baseline}

Of the 201 patients enrolled in the parent Proxima B study, 32 had unilateral GA at baseline (fellow eye iAMD cohort, referred to as the "eye of interest" in the present analyses). Mean patient age was 75.1 years, with a majority of patients aged $\leq 84$ years. All patients were white, and nearly $60 \%$ were female. Mean \pm standard deviation BCVA in the study eye at baseline was $56.6 \pm 19.5$ (approximate Snellen equivalent 20/80), and the number of eyes with BCVA worse than 20/100 and 20/40 or better was comparable [12]. Two patients were excluded from analysis due to nonavailability of follow-up data. Median observation period duration was 17.8 months, ranging from 10.1 to 31.9 months, with median interval time of 6 months between visits. Patient characteristics at baseline have been published previously [12].

\section{Conversion from iAMD to GA and Characteristics \\ of $G A$ Converters in the Eye of Interest (Proxima $B$ \\ Fellow Eye)}

Of the 30 eyes with available follow-up images analyzed, 12 converted to GA as assessed on FAF (according to the originally applied definition of GA as used in the parent Proxima B study and described above in Materials and Methods). Among converters to GA, mean total lesion area at first manifestation was $0.46 \mathrm{~mm}^{2}$, with the 


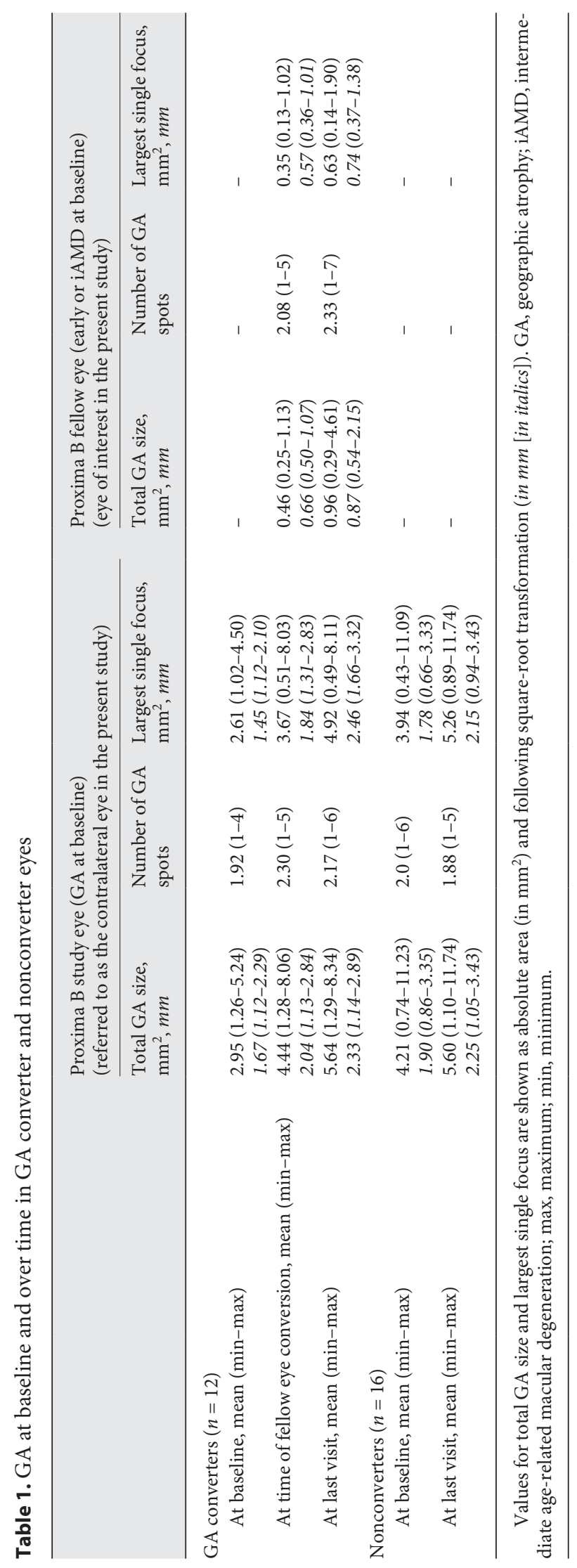

largest individual lesion ranging from 0.13 to $1.02 \mathrm{~mm}^{2}$ (Table 1). At the last visit, mean total lesion area in eyes converted to GA was $0.96 \mathrm{~mm}^{2}$, with the largest individual lesion ranging from 0.14 to $1.90 \mathrm{~mm}^{2}$. Comparing GA progression rates between contralateral eyes (originally study eyes in the parent Proxima B study) and eyes converting to GA in the present study, the latter exhibited a much faster growth rate versus the former, respectively ( 1.95 vs. $0.88 \mathrm{~mm}^{2} /$ year; Table 2$)$. Following square-root transformation, this difference was largely reduced $(0.44$ vs. $0.38 \mathrm{~mm} /$ year).

Two different clinical patterns were identified that led to GA conversion on FAF. In 6 eyes, GA development was spatially associated with large soft drusen, either with ( $n$ $=3)$ or without $(n=1)$ hyperpigmentary changes or with crystalline deposits $(n=2)$, as seen in the available multimodal longitudinal dataset. This pattern preceding atrophy manifestation showed previously described features of nGA, including subsidence of the INL and OPL and presence of a hyporeflective wedge-shaped band within Henle's fiber layer (shown in Fig. 1 and online suppl. Fig. 1; see www.karger.com/doi/10.1159/000517881 for all online suppl. material). In the remaining 6 eyes, GA occurred in local absence of large drusen and was associated with high density, high number of RPD lesions, and marked ONL thinning (shown in Fig. 2 and online suppl. Fig. 2). Initial occurrence of atrophy qualitatively encompassed larger areas with a more initial placoid atrophy appearance and was spatially confined to areas without any large druse. By FAF imaging, intensity within these early atrophic lesions appeared to be less decreased ("more grayish") versus severely reduced intensities seen at the site of large drusen-associated atrophy. Further, intensive focal areas of increased FAF signal were commonly seen surrounding RPD-associated atrophy development. Quantitative analysis confirmed these observations; initial mean GA area ( 0.57 vs. $0.35 \mathrm{~mm}^{2}$ ) was larger in RPD-associated versus drusen-associated GA development (Table 3). Where further GA progression data were available, RPD-associated GA $(n=4)$ exhibited a faster early lesion growth versus drusen-associated GA $(n=5$; 1.49 vs. $0.38 \mathrm{~mm}^{2} /$ year). Even after applying square-roottransformed GA enlargement rates, RPD-associated progression was more than double the rate of that associated with large drusen-associated atrophy ( 0.66 vs. $0.30 \mathrm{~mm} /$ year). Online suppl. Figures 3 and 4 provide representative images from nonconverter eyes. Online suppl. Figure 5 provides annualized growth rate for each patient and growth rate for converters/nonconverters based on RPD status. 
Table 2. GA progression in GA converter and nonconverter eyes

\begin{tabular}{|c|c|c|}
\hline & $\begin{array}{l}\text { Proxima B study eye (GA at } \\
\text { baseline) (referred to as the } \\
\text { contralateral eye in the present } \\
\text { study) }\end{array}$ & $\begin{array}{l}\text { Proxima B fellow eye (early } \\
\text { or iAMD at baseline) (eye } \\
\text { of interest in the present } \\
\text { study) }\end{array}$ \\
\hline & $\begin{array}{l}\text { Progression rate, } \\
\mathrm{mm}^{2} / \text { year, } \mathrm{mm} / \text { year }\end{array}$ & $\begin{array}{l}\text { Progression rate, } \\
\mathrm{mm}^{2} / \text { year, } \mathrm{mm} / \text { year }\end{array}$ \\
\hline From baseline to ET, mean (min-max) & $0.43(0.01-0.70)$ & \\
\hline \multirow[t]{2}{*}{ Once conversion occurred in fellow eye,${ }^{a}$ mean $(\min -\max )$} & $1.95(0.03-3.82)$ & $0.88(0.02-3.28)$ \\
\hline & $0.44(0.01-0.81)$ & $0.38(0.02-1.22)$ \\
\hline \multicolumn{3}{|l|}{ Nonconverters $(n=16)$} \\
\hline From baseline to ET, mean (min-max) & $1.02(0.14-3.63)$ & \\
\hline
\end{tabular}

Values are shown as absolute progression rate (in $\mathrm{mm}^{2} /$ year) and following square-root transformation (in $\mathrm{mm} /$ year [in italics]). ET, early termination; GA, geographic atrophy; iAMD, intermediate age-related macular degeneration; max, maximum; min, minimum. ${ }^{a}$ For $9 / 12$ converted fellow eyes, $\geq 1$ follow-up visit was available; therefore, extrapolated yearly progression rates are based on 9 eyes.

Features of iRORA and cRORA were identified on SDOCT in all eyes that progressed to GA on FAF at the same location in the fundus in a given eye. Median time from iRORA to cRORA was 7 months, and median time from cRORA to GA was also 7 months. All lesions that converted to GA had RPE disruption $\geq 250 \mu \mathrm{m}$ and signs of photoreceptor degeneration at baseline (i.e., 2/3 criteria for cRORA were present, while the third criterion, defined as hypertransmission $\geq 250 \mu \mathrm{m}$, was not always present at baseline). Figure 3 illustrates precursor lesions of iRORA and cRORA on SD-OCT to manifest GA on FAF for each converter.

\section{Conversion from iAMD to $M N V$}

Of the 30 eyes analyzed, 2 developed clear signs of MNV on multimodal imaging, 1 with predominantly RPD and a new intraretinal and subretinal hemorrhage, and 1 within a large area of drusen with subretinal and intraretinal fluid.

\section{Characteristics of Nonconverters}

Because 12 eyes converted to GA and 2 eyes converted to $\mathrm{MNV}$, a total of $16 / 30$ eyes with follow-up time showed no conversion to advanced AMD during the observation period, which was $>12$ months for 15 eyes and $<12$ months for only 1 eye. As shown in Table 1, at baseline, these nonconverters had both larger mean total GA and mean largest single focus size in the contralateral eye (originally study eyes in the parent Proxima B study) versus convert- ers. At the last visit, the mean total GA size and mean largest single focus size in these contralateral eyes were similar in nonconverters and converters (Table 1). In accordance, mean GA progression rates in these contralateral eyes, in both absolute area rates and following squareroot transformation, were higher in converters versus nonconverters ( 1.79 vs. $1.02 \mathrm{~mm}^{2} /$ year and 0.43 vs. 0.25 $\mathrm{mm}$ /year; Table 2).

Of the 16 eyes that did not convert to GA, 2 eyes had large central vitelliform lesions associated with pigment epithelium detachments (PEDs) at baseline and 3 eyes had predominantly RPD with no obvious large drusen. The remaining 11 eyes showed predominant drusenoid elevations. While 5/11 eyes appeared to be on the typical path of large drusenoid atrophy development, but had not reached the stage of manifest GA at early termination, there were 6 eyes with large, shallow drusenoid PEDs ("double-layer sign") at baseline that showed only subtle progression during the observation period (shown in Fig. 4). Of note, these eyes did not show any signs of exudation, such as intraretinal or subretinal fluid [19]. FA imaging at baseline did show faint hyperfluorescence as a staining phenomenon, with no clear signs of late-phase leakage. Representative images of clinical characteristics found in eyes of nonconverters are shown in Figure 4. Eleven nonconverters met criteria for iRORA at some point during their follow-up, and 4 met criteria for cRORA during their follow-up. 


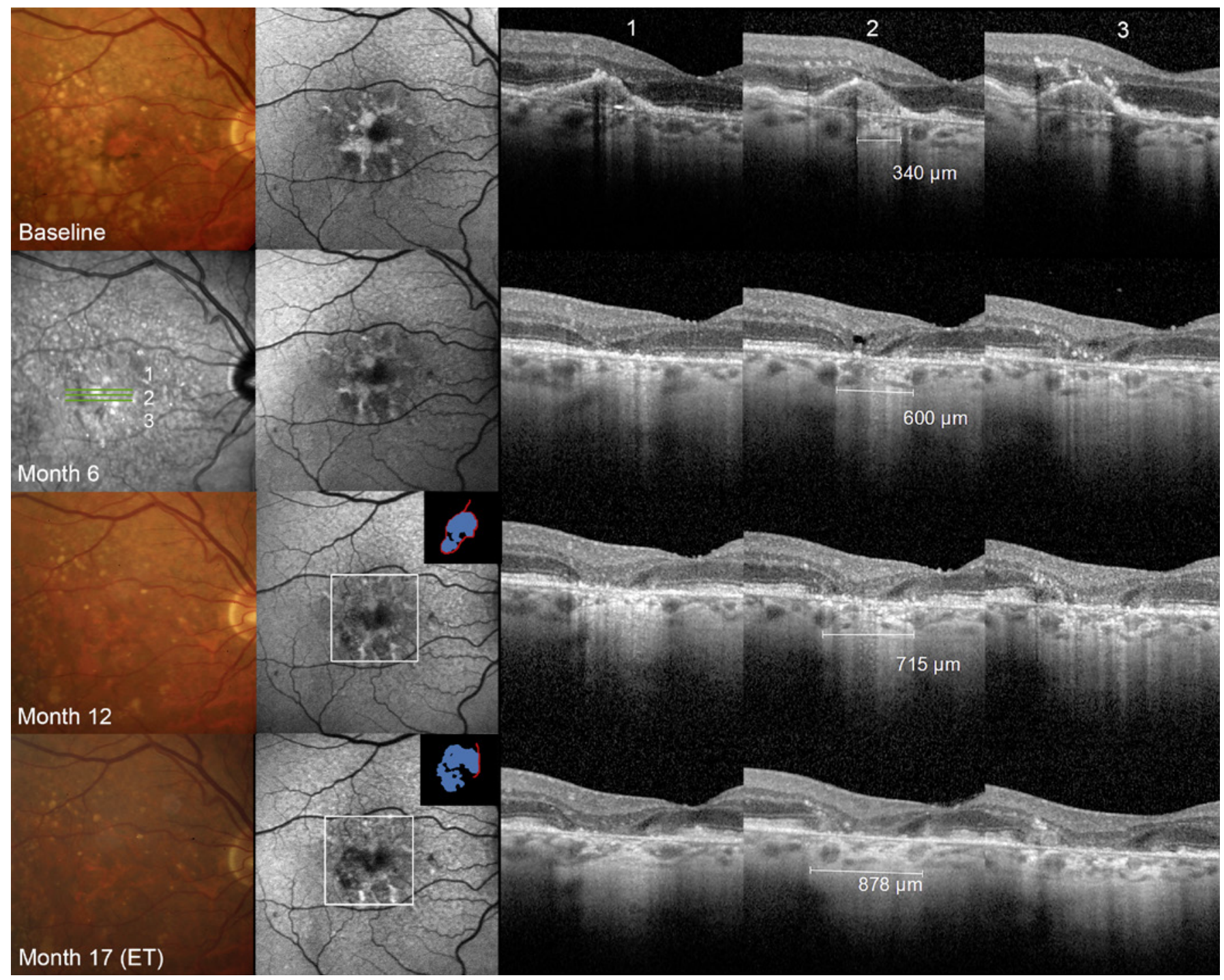

Fig. 1. Large drusen-associated atrophy development from iAMD. Images of the right eye with large drusen-associated atrophy development, demonstrated by (from left to right) $\mathrm{CFP}, \mathrm{FAF}$, and 3 representative neighboring SD-OCT B-scans for each visit (from top to bottom: baseline, month 6, month 12, and month 17 ET). Second row: CFP was not planned for 6-month interval visits. Instead, NIR images are shown here, also illustrating the locations of the 3 B-scans (from top: 1-3) by green color. At baseline (top row), hyperpigmentary changes along drusen and areas of increased FAF are present. The OCT scans show an area of a large druse with corresponding subsidence of the OPL and the INL, ONL thinning, and localized RPE thinning and thickening at the top of the druse. At baseline and at month 6, both the diameter of RPE disruption and the area of choroidal hypertransmission are $\geq 250 \mu \mathrm{m}$ (i.e., cRORA is present), along with loss of the ONL morphology. At month 6, a hyporeflective wedge-shaped band within Henle's fiber layer is also clearly visible. At month 12 , conversion to GA had been determined (as part of the original Proxima B definitions). The insert to the top right of the FAF image shows the annotated lesion (size: $0.491 \mathrm{~mm}^{2}$ ), followed by OCT signs of cRORA progression. At month 17, lesion enlargement by both FAF (lesion size: $0.668 \mathrm{~mm}^{2}$ ) and OCT is evident. CFP, color fundus photography; cRORA, complete retinal pigment epithelium and outer retinal atrophy; ET, early termination; FAF, fundus autofluorescence; GA, geographic atrophy; iAMD, intermediate age-related macular degeneration; INL, inner nuclear layer; NIR, near-infrared reflectance; OCT, optical coherence tomography; ONL, outer nuclear layer; OPL, outer plexiform layer; RPE, retinal pigment epithelium; SD-OCT, spectral-domain optical coherence tomography. 


\section{Subclinical Nonexudative MNV}

The original Proxima B study did not include OCT angiography (OCT-A) in the study protocol, and FA was only mandatory at baseline. In order to account for recent observations that nonexudative MNV is actually more common than previously anticipated in iAMD, and the observation of a high relative prevalence of shallow PEDs in the nonconverter subgroup, we looked for and identi- fied the presence of PEDs $\geq 1,000 \mu \mathrm{m}$ in diameter at baseline in $13 / 32$ eyes (Table 4 ). In $8 / 13$ eyes, there was presence of SIRE sign. In another 2 eyes, irregular RPE elevations were identified with the typical double-layer configuration, although diameter did not reach the 1,000$\mu \mathrm{m}$ criterion. In total, signs suggestive of nonexudative MNV were found in a total of 10 eyes by structural OCT imaging, of which 6 occurred in the nonconverter group.

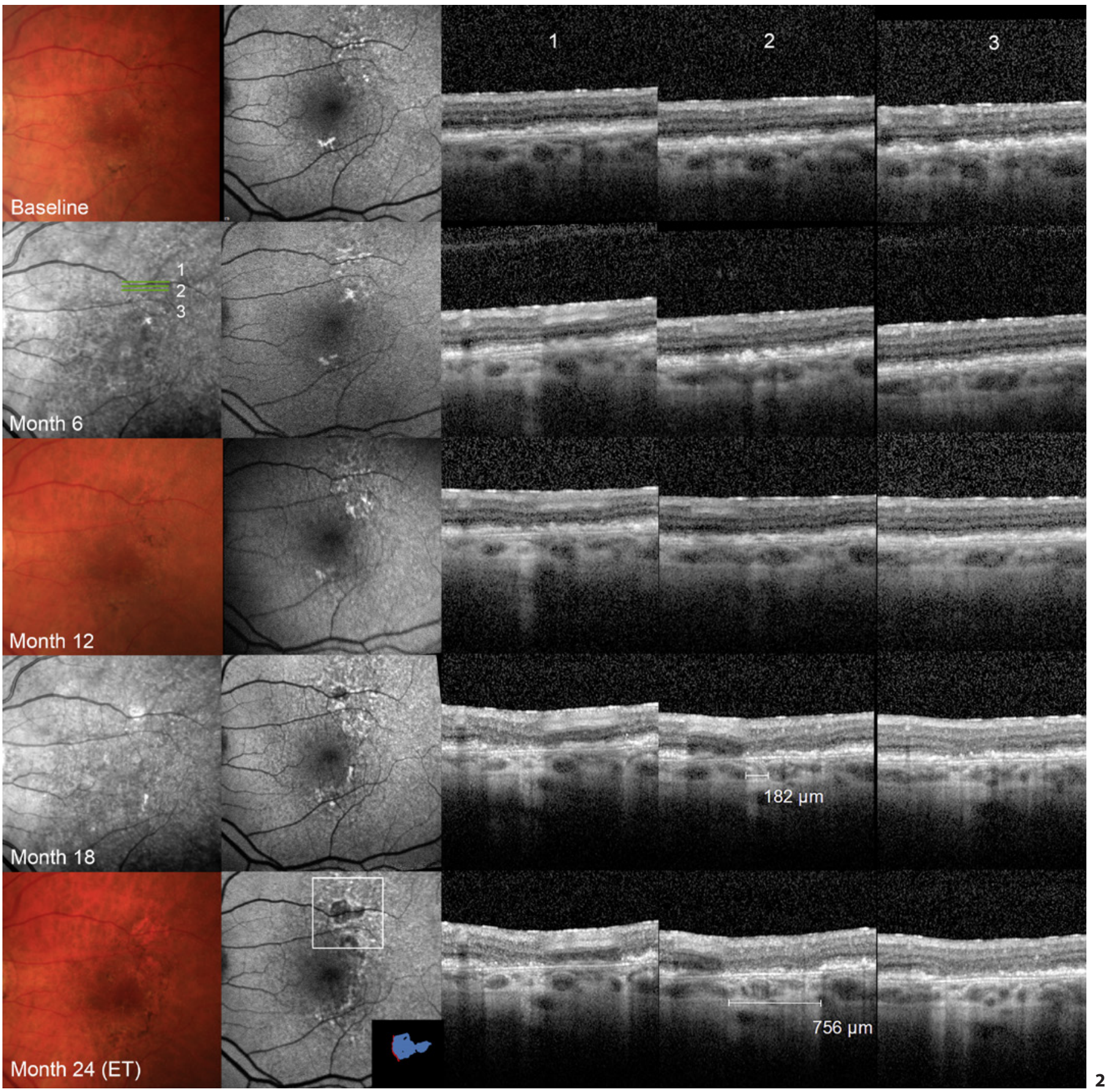

(For legend see next page.)

Multimodal Approach to Assess Progression to GA in the Proxima B Subcohort
Ophthalmologica 2021;244:523-534 DOI: $10.1159 / 00051788$ 
Table 3. Mean GA scores for converters at time of conversion from iAMD to GA in Proxima B fellow eyes (eyes of interest in the present study) for large drusen-associated atrophy and RPD-associated atrophy

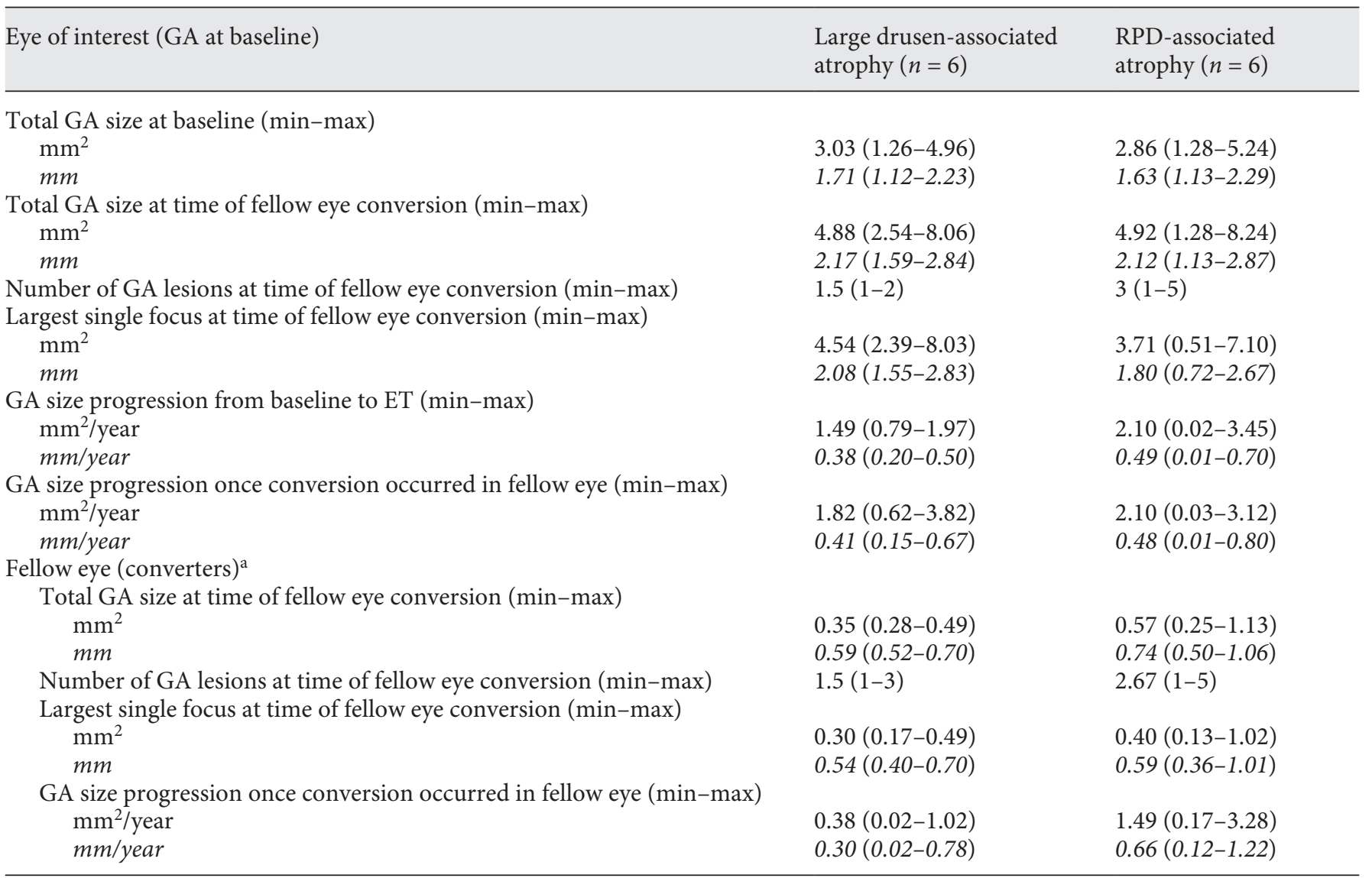

ET, early termination; GA, geographic atrophy; iAMD, intermediate age-related macular degeneration; max, maximum; min, minimum; RPD, reticular pseudodrusen. ${ }^{\text {a }}$ For $9 / 12$ converted fellow eyes, $\geq 1$ follow-up visit was available; therefore, extrapolated yearly progression rates are based on 9 eyes ( 5 for drusen-associated atrophy and 4 for RPD-associated atrophy).

Fig. 2. RPD-associated atrophy development from iAMD. Images of the left eye with RPD-associated atrophy development, demonstrated by (from left to right) $\mathrm{CFP}, \mathrm{FAF}$, and 3 representative neighboring SD-OCT B-scans for each visit (from top to bottom: baseline, month 6 , month 12 , month 18, and month 24 ET). Second row: CFP was not planned for 6-month and 18-month interval visits. Instead, NIR images are shown here, also illustrating the locations of the $3 \mathrm{~B}$-scans (from top: 1-3) by green color at the month 6 visit. At baseline (top row), no large drusen in the presence of RPD and areas of focal increased FAF are present. The OCT scans show severe ONL thinning and splitting of the RPE/Bruch's membrane band with thickening/hyperreflective material at the level of the RPE. Over time, progression of these OCT signs and larger areas of focally increased FAF are observed. At month 18, a small area of decreased FAF ("more grayish appearance") and choroidal hypertransmission with a diameter of $182 \mu \mathrm{m}$, along with RPE dis- ruption, are shown. These changes are at the site of a retinal blood vessel (superior to the fovea), making lesion size determination challenging. At month 24, conversion to GA had been determined (as part of the original Proxima B definitions). The insert to the bottom right of the FAF image shows the annotated lesion (size: $0.553 \mathrm{~mm}^{2}$ at the site of the retinal blood vessel superior to the fovea). At the same time, clear signs of RPE degeneration and choroidal hypertransmission $\geq 250 \mu \mathrm{m}$ in diameter are visible (i.e., cRORA). CFP, color fundus photography; cRORA, complete retinal pigment epithelium and outer retinal atrophy; ET, early termination; FAF, fundus autofluorescence; GA, geographic atrophy; iAMD, intermediate age-related macular degeneration; NIR, nearinfrared reflectance; OCT, optical coherence tomography; ONL, outer nuclear layer; RPD, reticular pseudodrusen; RPE, retinal pigment epithelium; SD-OCT, spectral-domain optical coherence tomography. 
Fig. 3. Evolution to GA at respective study visits. For each of the 12 converters, the stage in the evolution to atrophy at respective study visits is shown (top [filled symbols]: large drusen-associated atrophy; bottom [open symbols]: RPD-associated atrophy). End of study visits occurred between the prespecified 6-month intervals. cRO$\mathrm{RA}$, complete retinal pigment epithelium and outer retinal atrophy; FAF, fundus autofluorescence; GA, geographic atrophy; iAMD, intermediate age-related macular degeneration; iRORA, incomplete retinal pigment epithelium and outer retinal atrophy; M, month; RPD, reticular pseudodrusen; SCR, screening; SD-OCT, spectraldomain optical coherence tomography.

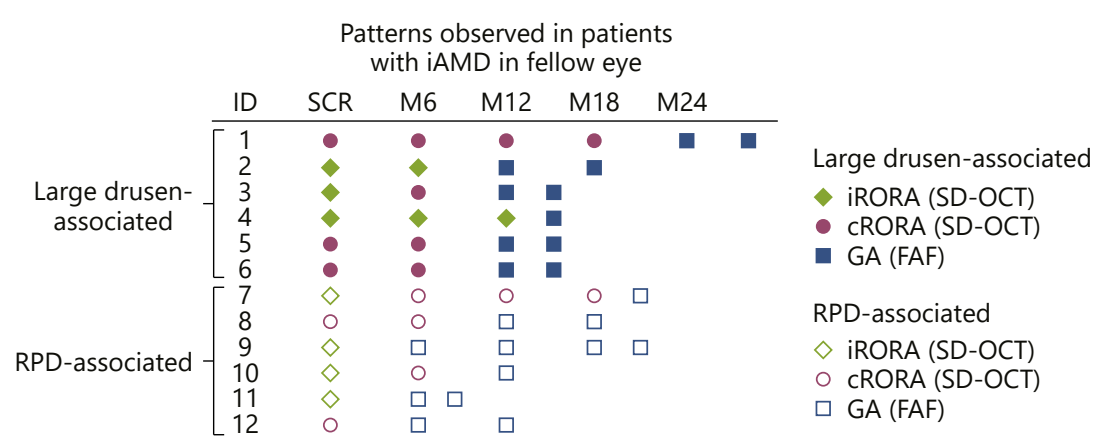

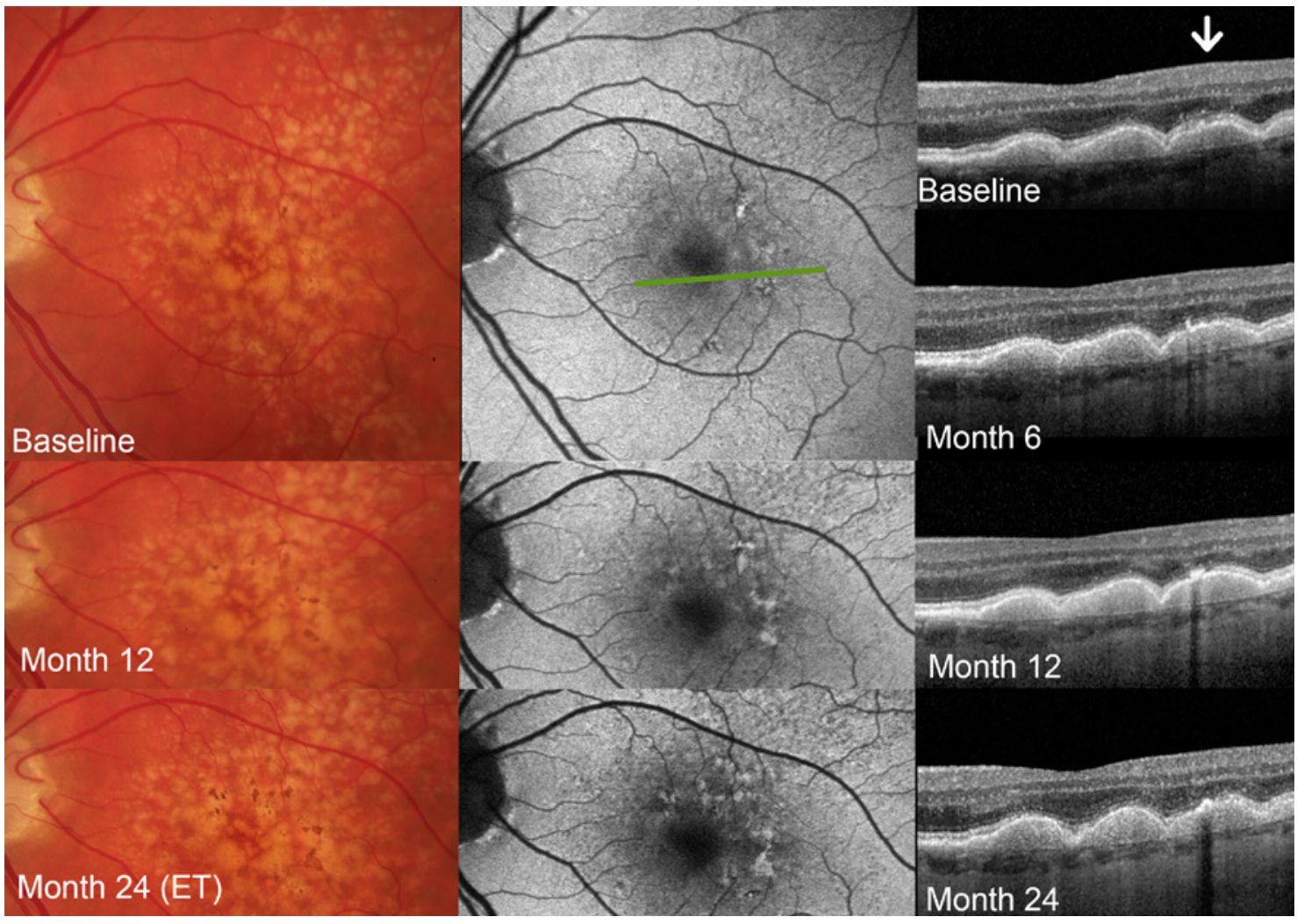

Fig. 4. Representative images from a nonconverter eye. Images of the left eye not showing conversion within the review period, demonstrated (from left to right) by CFP, FAF, and representative SDOCT B-scan for selected visits (from top to bottom: baseline, month 6 [SD-OCT only], month 12, and month 24 ET). The FAF image at baseline shows in green color the location of the B-scan. At baseline, hyperpigmentary changes by colors and areas of increased and decreased FAF are detected, which correspond to an area of confluent drusen by OCT. At the site of one of these druse (white arrow), ONL thinning and subtle disruption of the ELM and EZ are present. Over time, the amount of hyperpigmentary changes increases with increased FAF abnormalities, while drusen mor- phology shows only slight changes. From month 12 onward, localized RPE thickening with hyporeflectivity versus the choroid occurs. At all visits, no signs of choroidal hypertransmission were detected (i.e., no iRORA or cRORA present). CFP, color fundus photography; cRORA, complete retinal pigment epithelium and outer retinal atrophy; ELM, external limiting membrane; ET, early termination; EZ, ellipsoid zone; FAF, fundus autofluorescence; iRORA, incomplete retinal pigment epithelium and outer retinal atrophy; OCT, optical coherence tomography; ONL, outer nuclear layer; RPE, retinal pigment epithelium; SD-OCT, spectraldomain optical coherence tomography. 
Table 4. Assessment of OCT-suggestive signs of nonexudative MNV

\begin{tabular}{llll}
\hline & $\begin{array}{l}\text { General presence of } \\
\text { PED with lateral } \\
\text { diameter } \geq 1,000 \mu \mathrm{m}^{\mathrm{a}}\end{array}$ & $\begin{array}{l}\text { Presence of SIRE sign (PED } \\
\geq 1,000 \mu \mathrm{m} \text { in diameter and } \\
\leq 100 \mu \mathrm{m} \text { in height) }\end{array}$ & $\begin{array}{l}\text { Presence of shallow PED } \\
(\mathrm{PED} \geq 350 \mu \mathrm{m} \text { in diameter } \\
\text { and } \leq 100 \mu \mathrm{m} \text { in height) }\end{array}$ \\
\hline GA converters $(n=12)$ & 4 & 3 & 3 \\
MNV converters $(n=2)$ & 1 & 0 & 0 \\
Nonconverters $(n=16)$ & 7 & 4 & 6 \\
No follow-up $(n=2)$ & 1 & 1 & 1 \\
All eyes $(n=32)$ & 13 & 8 & 10 \\
\hline
\end{tabular}

GA, geographic atrophy; MNV, macular neovascularization; OCT, optical coherence tomography; PED, pigment epithelium detachment; SIRE, shallow, irregular retinal pigment epithelium elevations. ${ }^{\text {a }}$ Regardless of the height.

\section{Discussion}

This retrospective exploratory subgroup analysis of data from the Proxima B trial presents a multimodal imaging approach anchored on OCT imaging to identify and characterize precursors for development of GA in eyes with iAMD. In eyes that converted to GA during the review period, 2 patterns of GA development and progression were observed by multimodal imaging, 1 spatially associated with RPD, and 1 spatially associated with large soft drusen. GA lesion area progression was faster in those with RPD-associated GA development. In addition, patients with RPD had larger GA total lesion size at first manifestation (within prefixed 6-month intervals), suggesting more rapid development and progression of atrophic lesions.

In contrast to drusen, $\mathrm{RPD}$, associated with late stages of AMD, including type $3 \mathrm{MNV}$ and GA, are located in the subretinal space and may potentially extend up to ONLs. Their occurrence has been linked to genetic factors that predispose to AMD development, as well as to increased age of AMD onset and female sex [20]. Although the precise composition of RPD is not yet determined, they have been shown to be distinct from drusen and contain vitronectin and photoreceptor pigments, as well as microglia and macrophages, suggesting a role in inflammation $[16,21,22]$.

The association between RPD and late-stage AMD, particularly GA, has been investigated in multiple studies [23-25]. Conversion from iAMD to GA was observed to be more frequent in eyes with RPD than in those without RPD in a study of 126 eyes in 92 patients ( $74.2 \%$ vs. $41.7 \%$, respectively; $p<0.001$ ) [23]. In the large multicenter AgeRelated Eye Disease Study 2, among 5,021 eyes in 2,516 participants, RPD was noted in 1,186 (24\%) eyes. Relative risk of developing GA at the next annual visit was $2.42(p$
$<0.001$ ), and risk of developing neovascular AMD was $1.21(p=0.26)$. Thus, patients with RPD had a greater risk of progression to GA, but not to neovascular AMD [25].

In larger longitudinal $(n=181)$ and cross-sectional retrospective observational $(n=230)$ studies in patients with iAMD [26], nGA identified on SD-OCT as subsidence in the OPL and INL, and development of a hyporeflective wedge within the OPL, was correlated with development of drusen-associated atrophy. These features preceding atrophy, described as $\mathrm{nGA}$, were also observed in the 6 eyes with RPD that converted to GA in the current analyses. In another retrospective analysis $(n=21)$, a late form of AMD characterized by outer retinal atrophy and loss of underlying choroidal thickness occurred in eyes showing regression of subretinal drusenoid deposits [27], a symptom commonly observed in eyes with RPD. These observations further support a correlation between RPD and progression to GA. Ongoing prospective natural history studies, including the MACUSTAR study, address these associations in more detail in larger cohorts [28].

In our present analysis of patients with unilateral GA, comparing characteristics of the eye with GA at baseline, it was observed that total GA size tends to be higher in nonconverters. In addition, GA area progression was markedly slower in corresponding eyes of nonconverters than in converters, even following square-root transformation to compensate for smaller lesion sizes at presentation. In this context, it is interesting to note that in the majority of these patients, eyes of nonconverters in Proxima B, classified as having iAMD based on standard retinal imaging, had signs by structural OCT suggestive of nonexudative $\mathrm{MNV}$, including the previously described double-layer and SIRE signs [19]. Of note, while FA was mandatory at baseline, it was not required at subsequent visits, and OCT-A was not part of the examinations re- 
quired by study protocol. Since the conduct of Proxima $\mathrm{A}$ and $\mathrm{B}$, understanding of nonexudative MNV manifestations has evolved, particularly based on more recent observations from OCT-A assessments [29]. Recent reports confirm previous anecdotal observations that GA lesion size progression may be slower when $\mathrm{MNV}$ is present in parallel [30]. These differences have recently been quantified [31]. Overall, observations in this study may support the concept that nonexudative MNV has protective effects on atrophy development and progression, although the number of cases is small [32].

Limitations of this study include that it was a retrospective subgroup analysis of a larger clinical trial, and participant sample size was small, with only 30 patients with available follow-up data. As a result, analyses are descriptive and, therefore, no statistical analyses were performed.

\section{Conclusion}

Identification of precursor lesions and characteristics of GA occurrence and growth will be important for development of future therapeutic approaches in iAMD. This hypothesis-generating analysis specifically suggests that details on characteristic pathophysiological features of lesion growth in GA, such as presence of RPD, identification of iRORA/cRORA, and recognition of signs for nonexudative MNV acquired through multimodal imaging, may be beneficial in selecting clinical trial end points. Furthermore, these precursor lesions may help identify therapeutic targets for future research on treatment of GA.

\section{Acknowledgments}

Funding was provided by F. Hoffmann-La Roche Ltd. for thirdparty writing assistance, which was provided by Priyanka Narang, PhD, of Envision Pharma Group. F. Hoffmann-La Roche Ltd., Basel, Switzerland, provided financial support for the study and participated in the study design; conducting the study; data collection, management, analysis, and interpretation; and preparation, review, and approval of the manuscript.

\section{Statement of Ethics}

Not applicable to the present analysis. The parent study, Proxima B, was approved by the institutional review board and received ethics committee approval at each participating site. The present study is a retrospective analysis of the Proxima B study (Holekamp N, Wykoff CC, Schmitz-Valckenberg S, Monés JM, Souied EH, Lin
$\mathrm{H}$, et al. Natural history of geographic atrophy secondary to agerelated macular degeneration: results from the prospective Proxima $A$ and B clinical trials. Ophthalmology. 2020 Jun;127(6):769-83).

\section{Conflict of Interest Statement}

Steffen Schmitz-Valckenberg receives financial support from Acucela/Kubota Vision, Bayer, Carl Zeiss Meditec, CenterVue, Genentech, Inc./Roche, Heidelberg Engineering, Katairo, and Optos; serves as a consultant for and receives financial support from Alcon/Novartis, Allergan, and Bioeq/Formycon; and as a consultant for Apellis, Galimedix, and Oxurion. Martina D. Braun and Marlene Saßmannshausen receive financial support from Carl Zeiss Meditec, CenterVue, and Heidelberg Engineering. Sarah Thiele received financial support from Bayer, Bonfor Gerok Funding (Faculty of Medicine, University of Bonn Grant No. O-137.0026), Carl Zeiss Meditec, CenterVue, Heidelberg Engineering, Novartis, and Optos. Daniela Ferrara, Lee Honigberg, Simon S. Gao, Hao Chen, and Verena Steffen are employees of Genentech, Inc. Daniela Ferrara reports stocks/stock options from Roche. Frank G. Holz serves as a consultant for and receives financial support from Acucela, Apellis, Bayer, Bioeq/Formycon, Chengdu Kanghong, Genentech, Inc./Roche, Graybug, Heidelberg Engineering, Lin BioScience, Oxurion, Pixium, and Stealth; receives financial support from Allergan, and Zeiss; and serves as a consultant for Boehringer Ingelheim, CenterVue, Ellex, Geuder, NightstaRx, and Optos.

\section{Funding Sources}

This study was supported in part by an unrestricted grant from Research to Prevent Blindness, New York, NY, USA, to the Department of Ophthalmology \& Visual Sciences, University of Utah, Salt Lake City, UT, USA.

\section{Author Contributions}

All authors contributed to the planning, conduct, and reporting of the work described in the article. All authors had full access to all the data in the study and take responsibility for the integrity of the data and the accuracy of the data analysis.

\section{Data Availability Statement}

Qualified researchers may request access to individual patientlevel data through the clinical study data request platform (https:// vivli.org/). Further details on Roche's criteria for eligible studies are available here (https://vivli.org/members/ourmembers/). For further details on Roche's Global Policy on the Sharing of Clinical Information and how to request access to related clinical study documents, see here (https://www.roche.com/research_and_development/who_we_are_how_we_work/clinical_trials/our_commitment_to_data_sharing.htm). 


\section{References}

1 Wong WL, Su X, Li X, Cheung CM, Klein R, Cheng CY, et al. Global prevalence of age-related macular degeneration and disease burden projection for 2020 and 2040: a systematic review and meta-analysis. Lancet Glob Health. 2014 Feb;2(2):e106-16.

2 Flaxman SR, Bourne RRA, Resnikoff S, Ackland $\mathrm{P}$, Braithwaite $\mathrm{T}$, Cicinelli MV, et al. Global causes of blindness and distance vision impairment 1990-2020: a systematic review and meta-analysis. Lancet Glob Health. 2017 Dec;5(12):e1221-34.

3 Arya M, Sabrosa AS, Duker JS, Waheed NK. Choriocapillaris changes in dry age-related macular degeneration and geographic atrophy: a review. Eye Vis. 2018;5:22.

4 Wu Z, Luu CD, Hodgson LAB, Caruso E, Tindill N, Aung KZ, et al. Prospective longitudinal evaluation of nascent geographic atrophy in age-related macular degeneration. Ophthalmol Retina. 2020 Dec 16;4(6):568-75.

5 Sarks JP, Sarks SH, Killingsworth MC. Evolution of geographic atrophy of the retinal pigment epithelium. Eye. 1988;2(Pt 5):552-77.

6 Sunness JS, Gonzalez-Baron J, Applegate CA, Bressler NM, Tian Y, Hawkins B, et al. Enlargement of atrophy and visual acuity loss in the geographic atrophy form of age-related macular degeneration. Ophthalmology. 1999 Sep;106(9):1768-79.

7 Lindblad AS, Lloyd PC, Clemons TE, Gensler GR, Ferris FL 3rd, Klein ML, et al. Change in area of geographic atrophy in the Age-Related Eye Disease Study: AREDS report number 26. Arch Ophthalmol. 2009 Sep;127(9):1168-74.

8 Smailhodzic D, Fleckenstein M, Theelen T, Boon CJ, van Huet RA, van de Ven JP, et al. Central areolar choroidal dystrophy (CACD) and age-related macular degeneration (AMD): differentiating characteristics in multimodal imaging. Invest Ophthalmol Vis Sci. 2011 Nov;52(12):8908-18.

9 Holz FG, Bindewald-Wittich A, Fleckenstein M, Dreyhaupt J, Scholl HP, Schmitz-Valckenberg S. Progression of geographic atrophy and impact of fundus autofluorescence patterns in age-related macular degeneration. Am J Ophthalmol. 2007 Mar;143(3):463-72.

10 Schmitz-Valckenberg S, Sahel JA, Danis R, Fleckenstein M, Jaffe GJ, WolfS, et al. Natural history of geographic atrophy progression secondary to age-related macular degeneration (Geographic Atrophy Progression study). Ophthalmology. 2016 Feb;123(2): 361-8.

11 Keenan TD, Agrón E, Domalpally A, Clemons TE, van Asten F, Wong WT, et al. Progression of geographic atrophy in age-related macular degeneration: AREDS2 report number 16. Ophthalmology. 2018 Dec;125(12): 1913-28.
12 Holekamp N, Wykoff CC, Schmitz-Valckenberg S, Monés J, Souied EH, Lin H, et al. Natural history of geographic atrophy secondary to age-related macular degeneration: results from the prospective Proxima A and B clinical trials. Ophthalmology. 2020 Jun;127(6):76983.

13 Holz FG, Sadda SR, Busbee B, Chew EY, Mitchell P, Tufail A, et al. Efficacy and safety of lampalizumab for geographic atrophy due to age-related macular degeneration: Chroma and Spectri phase 3 randomized clinical trials. JAMA Ophthalmol. 2018 Jun 1;136(6):66677.

14 Schmitz-Valckenberg S, Brinkmann CK, Alten F, Herrmann P, Stratmann NK, Göbel AP, et al. Semiautomated image processing method for identification and quantification of geographic atrophy in age-related macular degeneration. Invest Ophthalmol Vis Sci. 2011 Sep;52(10):7640-6.

15 Suzuki M, Sato T, Spaide RF. Pseudodrusen subtypes as delineated by multimodal imaging of the fundus. Am J Ophthalmol. 2014 May; 157(5):1005-12.

16 Rabiolo A, Sacconi R, Cicinelli MV, Querques L, Bandello F, Querques G. Spotlight on reticular pseudodrusen. Clin Ophthalmol. 2017;11:1707-18

17 Sadda SR, Guymer R, Holz FG, SchmitzValckenberg S, Curcio CA, Bird AC, et al. Consensus definition for atrophy associated with age-related macular degeneration on OCT: Classification of Atrophy report 3. Ophthalmology. 2018 Apr;125(4):537-48.

18 Guymer RH, Rosenfeld PJ, Curcio CA, Holz FG, Staurenghi G, Freund KB, et al. Incomplete retinal pigment epithelial and outer retinal atrophy in age-related macular degeneration: Classification of Atrophy meeting report 4. Ophthalmology. 2019 Sep;127(3):394-409.

19 Narita C, Wu Z, Rosenfeld PJ, Yang J, Lyu C, Caruso E, et al. Structural OCT signs suggestive of subclinical nonexudative macular neovascularization in eyes with large drusen. Ophthalmology. 2020 May;127(5):637-47.

20 Boddu S, Lee MD, Marsiglia M, Marmor M, Freund KB, Smith RT. Risk factors associated with reticular pseudodrusen versus large soft drusen. Am J Ophthalmol. 2014 May; 157(5): 985-93.e2.

21 Greferath U, Guymer RH, Vessey KA, Brassington K, Fletcher EL. Correlation of histologic features with in vivo imaging of reticular pseudodrusen. Ophthalmology. 2016 Jun; 123(6):1320-31.

22 Wightman AJ, Guymer RH. Reticular pseudodrusen: current understanding. Clin Exp Optom. 2019 Sep;102(5):455-62.
23 Marsiglia M, Boddu S, Bearelly S, Xu L, Breaux BE Jr, Freund KB, et al. Association between geographic atrophy progression and reticular pseudodrusen in eyes with dry agerelated macular degeneration. Invest Ophthalmol Vis Sci. 2013 Nov;54(12):7362-9.

24 Finger RP, Chong E, McGuinness MB, Robman LD, Aung KZ, Giles G, et al. Reticular pseudodrusen and their association with agerelated macular degeneration: the Melbourne Collaborative Cohort Study. Ophthalmology. 2016 Mar;123(3):599-608.

25 Domalpally A, Agrón E, Pak JW, Keenan TD, Ferris FL 3rd, Clemons TE, et al. Prevalence, risk, and genetic association of reticular pseudodrusen in age-related macular degeneration: Age-Related Eye Disease Study 2 report 21. Ophthalmology. 2019 Dec;126(12):165966.

26 Wu Z, Luu CD, Ayton LN, Goh JK, Lucci LM, Hubbard WC, et al. Optical coherence tomography-defined changes preceding the development of drusen-associated atrophy in age-related macular degeneration. Ophthalmology. 2014 Dec;121(12):2415-22.

27 Spaide RF. Outer retinal atrophy after regression of subretinal drusenoid deposits as a newly recognized form of late age-related macular degeneration. Retina. 2013 Oct; 33(9):1800-8.

28 Terheyden JH, Finger RP, Schmitz-Valckenberg S, Agostini H, Dahlke C, Kuehlewein L, et al. [Development and validation of novel clinical endpoints in intermediate age-related macular degeneration in MACUSTAR]. Ophthalmologe. 2019 Dec;116(12):1186-93.

29 de Oliveira Dias JR, Zhang Q, Garcia JMB, Zheng F, Motulsky EH, Roisman L, et al. Natural history of subclinical neovascularization in nonexudative age-related macular degeneration using swept-source OCT angiography. Ophthalmology. 2018 Feb;125(2):25566.

30 Heiferman MJ, Fawzi AA. Progression of subclinical choroidal neovascularization in agerelated macular degeneration. PLoS One. 2019 Jun;14(6):e0217805.

31 Pfau M, Möller PT, Künzel SH, von der Emde $\mathrm{L}$, Lindner M, Thiele S, et al. Type 1 choroidal neovascularization is associated with reduced localized progression of atrophy in age-related macular degeneration. Ophthalmol Retina. 2020;4(3):238-48.

32 Chen L, Messinger JD, Sloan KR, Swain TA, Sugiura Y, Yannuzzi LA, et al. Nonexudative macular neovascularization supporting outer retina in age-related macular degeneration: a clinicopathologic correlation. Ophthalmology. 2020 Jul;127(7):931-47. 